\title{
Association of Insulin Therapy with Depression, Cognition and Quality of Life in Type 2 Diabetes Patients: A Cross-sectional Study
}

\author{
Rizwana Parveen ${ }^{1}$, Prem Kapur², Nidhi Bharal Agarwal 3,* \\ 'Pharmaceutical Medicine, Department of Pharmacology, School of Pharmaceutical Education and Research, Jamia Hamdard, New Delhi, INDIA. \\ ${ }^{2}$ Hamdard Institute of Medical Sciences and Research, HAH Centenary Hospital, Jamia Hamdard, New Delhi, INDIA. \\ ${ }^{3}$ Centre for Translational and Clinical Research, School of Chemical and Life Sciences, Jamia Hamdard, New Delhi, INDIA.
}

\begin{abstract}
Background: Type 2 diabetes mellitus (T2DM) is linked to a significant rate of depression and cognitive impairment, according to previous studies. However, limited research has been done on the effects of diabetes treatment on depression, cognitive function, or overall quality of life (OoL). This planned study investigated into depression, cognitive function, and quality of life in T2DM patients, as well as its relationship to treatment received. To study the association of insulin therapy with depression, cognition and quality of life in T2DM patients. Methods: This was a crosssectional study that included 300 participants, comprising of 150 T2DM patients and 150 healthy individuals. The Patient health questionnaire and the Mini-mental state examination were used to measure depression and cognitive function, respectively. QoL was assessed using the Short-Form 36 (SF-36) questionnaire. Based on the type of treatment, all of the patients were divided into two subgroups: oral hypoglycemic medications (OHAs) and insulin. Results: The study included 300 participants, including T2DM patients $(n=150)$ and healthy controls $(n=150)$. In individuals with T2DM, the chances of mild (aOR 2.21, $95 \%$ Ci 1.16-4.20; $p=0.016$ ) and severe (aOR 4.32, $95 \%$ Ci 1.27-14.67; $p=0.019$ ) depression was higher. T2DM
\end{abstract}

patients were also more likely to have cognitive impairment (aOR 2.86, $95 \% \mathrm{Ci} 1.16-6.99 ; p=0.021)$. Patients taking insulin had a substantially increased risk of depression (OR 2.127, $95 \% \mathrm{Ci}$ 1.070-4.228, $p=0.036$ ) Insulin-treated patients exhibited considerably poorer OoL ratings. Conclusion: The research illustrates that insulin therapy has a negative impact on depression and $\mathrm{QoL}$ in T2DM patients. As a result, it is recommended that insulin-treated T2DM patients require psychological assistance. In such cases, it is advised that measures to enhance QoL be implemented.

Key words: Depression, Cognitive impairment, Quality of life, Hypoglycemic, Insulin, Type 2 diabetes.

\section{Correspondence}

Dr. Nidhi Bharal Agarwal,

Centre for Translational and Clinical Research, School of Chemical and Life Sciences, Jamia Hamdard, New Delhi-110062, INDIA.

Email id: nidhi.bharal@gmail.com

DOI: 10.5530/ijpi.2021.3.58

\section{INTRODUCTION}

Diabetes mellitus is the most prevalent metabolic disease, ${ }^{1}$ causing end-organ damage in almost all vital organ, including the brain. ${ }^{2}$ As per reports from the International Diabetes Federation, 463 million adults had diabetes in 2019, which is estimated to reach 700 million by $2045 .{ }^{3}$ Patients' life expectancy has improved as medical amenities and treatment techniques have advanced. As a result, the complications of diabetes, as well as its treatment and psychological components, may have a negative impact on a variety of aspects of life, including quality of life (QoL). ${ }^{4}$ Depression and type 2 diabetes mellitus (T2DM) are important worldwide public health issues, and both are expected to be among the top five primary causes of disease burden by 2030.5 Emerging data supports an epidemiological link between depression and T2DM, although the reason is unknown ${ }^{6}$ and assumed to be multifactorial. ${ }^{7}$ Comorbidity is thought to be caused by a variety of characteristics of the disease, including psychological and psychosocial consequences. ${ }^{8}$ Despite the significant incidence and impact of comorbid depression in T2DM patients, there is no evidence that $\mathrm{T} 2 \mathrm{DM}$ treatment regimens reduce the risk of depression. ${ }^{9}$ T2DM patients taking oral therapy had a greater prevalence of depression, according to a cross-sectional research, ${ }^{10}$ whereas Joseph et al. found a substantial link between insulin usage and depression. ${ }^{11}$ In T2DM patients, however, a cohort study found no link between depression and therapy type. Previous research have found inconsistencies in the relationship between depression and therapy type, necessitating further research.

Diabetes is linked to decreased levels of cognitive function, according to an increasing amount of research, and may be a risk factor for mild cognitive impairment (MCI). Diabetes is linked to a 1.2-fold increase in the risk of cognitive impairment, according to research. ${ }^{12}$ There are few research evaluating the effect of diabetes treatment on cognitive performance, despite the fact that numerous studies have focused at the influence of diabetes on cognitive function. ${ }^{13}$ Antidiabetics are thought to enhance cognitive function in diabetics by addressing vascular and neurodegenerative problems, as well as via direct pharmacological characteristics such as anti-inflammatory actions. ${ }^{14}$ Clinical research, on the other hand, has shown conflicting outcomes. ${ }^{15,16}$ The significant risk of MCI in diabetic individuals highlights the necessity to monitor the predictors of these diseases and develop treatment options.

In the recent decade, the diabetes treatment emphasis has changed. Current diabetes treatment goals include not just metabolic management and the avoidance of acute and chronic consequences, but also the maintenance of a high quality of life. Diabetics have a poor quality of life, according to several clinical research. Diabetics have a poorer quality of life than healthy people, according to a case-control research. ${ }^{17}$ Furthermore, numerous cross-sectional studies have shown that diabetics

This is an open access article distributed under the terms of the Creative Commons Attribution-NonCommercial-ShareAlike 4.0 License, which allows others to remix, tweak, and build upon the work non-commercially, as long as the author is credited and the new creations are licensed under the identical terms. 
have poorer QoL ratings. ${ }^{18-20}$ Because research on the link between different treatment regimens for type 1 diabetes (T1DM) and type 2 diabetes (T2DM) patients and QoL have not been consistent, it is essential to assess the correlations between these parameters. ${ }^{21,22}$ In light of the foregoing, the current study's goal was to determine the prevalence of depression, cognitive impairment, and quality of life in T2DM patients. Furthermore, the impact of treatment type on depression, cognitive function, and QoL was studied.

\section{MATERIALS AND METHODS}

\section{Study Population}

The research was carried out in a tertiary care hospital. Males and females aged between 19 and 65 participated in the study. T2DM patients were included as cases. Control subjects were healthy individuals. The patients with T2DM included in the study. The patients with T1DM, history of severe psychiatric disorders, substance abuse, already taking any psychotropic drug, diabetes complications, hypertension, liver disease, renal disease, primary hyperparathyroidism, cancer, human immunodeficiency syndrome, obesity, pregnant women, taking Vitamin-D supplement, and unwilling to give written informed consent were all excluded. Based on the kind of treatment, all of the patients were divided into two sub-groups: oral hypoglycemic medications (OHAs) and insulin. Combinations of sulfonylureas (glimepiride, glipizide), biguanides (metformin), and dipeptidyl peptidase- 4 inhibitors were used in the OHAs (teneligliptin, denagliptin, linagliptin, saxagliptin, sitagliptin and vidagliptin). Patients who were given a combination of OHAs and insulin were included in the insulin group.

Six hundred and seven people were approached for participation, including 393 diabetes patients who visited the Diabetic Clinic and Medicine OPD. A total of 214 healthy people were screened for participation during regular checkups. Written Informed consent was obtained from all individual participants included in the study.

\section{Sample size calculation}

The optimum sample size was calculated based on the following formula:

$$
n=Z_{2}^{a} \frac{P Q}{L^{2}}
$$

Where $\mathrm{P}=$ Prevalence of type- 2 diabetes $=10 \%$

$\mathrm{Q}=100-\mathrm{P}=100-10=90 \% ; \mathrm{L}=$ Permissible error

Furthermore, the sample size was determined using the aforementioned formula, assuming 5\% permissible error, 5\% type one error, and $90 \%$ power. The operational sample size was estimated to be around 150 cases. Similarly, for statistical comparisons, an equal number of controls were included.

\section{Participant Characteristics}

The individuals' demographic and clinical data were recorded using a standard manner. Age, height, weight, history of alcohol or cigarette use, physical activity, dietary habits, sun exposure, educational level, diabetes duration, and current diabetic medication were among the data collected. Each subject's BMI was determined using their assessed weight and height. Cases' fasting plasma glucose and HbAlc levels were also recorded. The healthy individuals' available medical prescriptions and laboratory reports from their routine health examination were used to validate their eligibility for enrollment.

\section{Assessment of depression}

The PHQ-9 Patient Health Questionnaire was used to measure depression. PHQ-9 is a simple to use and self-administered questionnaire. It's a short questionnaire that rates the nine DSM-IV depression criteria on a scale of 0 (not at all) to 3 (very) (nearly every day). After receiving informed consent at the initial session, the individuals completed the PHQ-9 questionnaire. The PHQ-9 is used to diagnose depression and is scored as follows:

- 0-4 - no depression;

- 5-9 - mild depression

- 10-14- moderate depression

- 15-19- moderately severe depression

- 20-27- severe depression

\section{Assessment of cognitive function}

The Mini-Mental State Exam (MMSE) or the Hindi Mental State Examination were used to test cognitive function. On the day of the patient's enrolment, a cognitive evaluation was done. The MMSE is a 30-point scale with seven categories: orientation to location, orientation to time, registration, attention and concentration, recollection, language, and visual creation. The MMSE score is a number between 0 and 30, with higher values signifying greater performance. No cognitive impairment is indicated by a score of 24-30, mild cognitive impairment is indicated by a score of 18-23, and severe cognitive impairment is indicated by a score of $0-17$.

\section{Assessment of Quality of life}

The Short Form-36 (SF-36), one of the most widely used measures for measuring clinical findings, was utilized to assess quality of life. Physical Functioning (10), Social Functioning (2), Mental Health (5), Vitality (4), Role-Physical (4), Role-Emotional (3), Bodily Pain-intensity and interference in daily work- (2), General Health (2), Physical Functioning (10), Social Functioning (2), Mental Health (5), Vitality (4), Role-Physical (4), Role-Emotional (3), Role-Physical (4), Role-Emotional (3), Bo (6). The patient's quality of life was assessed on the day of enrolment.

Ethical Clearance: Ethical Clearance was obtained from the Jamia Hamdard institutional ethics committee prior to the commencement of the study.

\section{Statistical analysis}

There were both continuous and categorical variables in the data. As a result, quantitative variables are represented in terms of mean \pm standard deviation (SD). The Kolmogorov-Smirnov and Shapiro-Wilk tests were used to determine the normality of the continuous variables. The Student's $\mathrm{t}$ test or the Mann-Whitney $\mathrm{U}$ test were used to examine the relationship between two continuous variables. To examine differences in the frequencies of categorical variables, the $\chi^{2}$ and Fisher exact tests were utilized. The combined connections were investigated using multivariable logistic regression analysis. Logistic regression was used to calculate adjusted odds ratios (ORs). ANOVA was used to compare the groups' demographics, depression, cognitive performance, and QoL. Tuckey's test was also used for post hoc analysis to determine the precise significant groupings. The level of significance for all statistical tests was set at a two-sided $p$-value of 0.05 . All statistical analyses were carried out using SPSS 22 software.

\section{RESULTS}

A total of 300 subjects were included. The study comprised two groups: cases (patients diagnosed with T2DM) and controls (healthy individuals). Thus, 150 subjects in each group were included;

Out of 300 subjects, 145 (48.3\%) were females and 155 (51.7\%) were males. The mean $\pm \mathrm{SD}$ age of cases and controls was $45.89 \pm 6.80$ and $47.19 \pm 8.32$ years, respectively. Cases had a known T2DM of 
Table 1: Demographics of cases and controls.

\begin{tabular}{ccc}
\hline Characteristic & Cases $(n=150)$ & $\begin{array}{c}\text { Controls } \\
(n=150)\end{array}$ \\
\hline Age (years) & $47.19 \pm 8.32$ & $45.89 \pm 6.80$ \\
Sex & $72(48)$ & $73(48.7)$ \\
Female & $78(52)$ & $77(51.3)$ \\
Male & $25.41 \pm 2.74$ & $24.41 \pm 2.98$ \\
BMI (kg/m $\left.{ }^{2}\right)$ & & \\
Education & $50(33.3)$ & $19(12.7)$ \\
No Education & $100(66.6)$ & $131(87.3)^{*}$ \\
Educated & & $64(42.7)$ \\
Employment & $71(47.3)$ & $86(57.3)$ \\
Unemployed & $79(52.7)$ & $97.53 \pm 8.68^{*}$ \\
Employed & $171.79 \pm 69.83$ & - \\
FPG (mg/dL) & $4.62 \pm 5.41$ & - \\
Duration of diabetes (years) & $8.87 \pm 2.36$ & - \\
HbAlc (\%) & & - \\
Diabetes treatment & $98(65.33)$ & \\
OHAs & $52(34.67)$ & \\
Insulin & &
\end{tabular}

${ }^{\star} P<0.0001$, Data presented as Mean \pm SD or $\mathrm{n}(\%), \mathrm{n}$, number; BMI, body mass index; FPG, fasting plasma glucose; HbA1c, glycated hemoglobin; OHAs, oral hypoglycemic agents. mean duration $4.62 \pm 5.41$ years. The BMI of cases was significantly higher than those of controls, $25.41 \pm 2.74$ and $24.41 \pm 2.98(p=0.04)$, respectively (Table 1 ).

The characteristics of the subjects in the three subgroups (controls, oral hypoglycemic agents (OHAs) group and insulin group) is shown in the Table 2.

Assessment of depression in cases and controls - The mean PHQ 9 score for cases was $4.74 \pm 6.52$, whereas for controls it was lower, $1.75 \pm 4.15$, $p=0.000$. The prevalence of depression was significantly higher in the cases than in the controls $(60(40 \%)$ vs. 25 (16.7\%), $p=0.000)$. In multivariate logistic regression, the odds of having mild depression in patients with T2DM was found to be more than twice than that in the control subjects (aOR 2.21, 95\% CI 1.16-4.20; $p=0.016$ ). Additionally, the odds of having severe depression in cases was also higher than in the controls (aOR 4.32, 95\% CI 1.27-14.67; $p=0.019$ ) (adjusted for age, sex, BMI, education and occupation). Among the total 85 (28.33\%) subjects having depression, a statistically higher prevalence of depression was observed among women $54(63.53 \%)$ than in men 31 (36.47\%), $p=0.003$. Of these 85 depressed subjects, unemployed subjects had higher prevalence of depression (51 (60\%) than those who were employed (34 (40\%)), $p=0.01$. In both groups it was observed that minimal/mild depression was more prevalent than moderate/severe depression (64 (21.33\%) and $21(7 \%)$, respectively). Numerically higher levels of HbAlc was observed in individuals with depression $(9.18 \pm 2.31)$ than those without depression (8.67 \pm 2.39$)$, however, the difference was statistically insignificant. Moreover, no significant difference was observed in the fasting plasma glucose (FPG) levels of individuals with depression and without depression.

Table 2: Characteristics of the subjects according to the type of treatment.

\begin{tabular}{|c|c|c|c|c|c|}
\hline Characteristic & & Controls $(n=150)$ & Oral hypoglycemic agents ( $n=98)$ & Insulin $(n=52)$ & $P$ value \\
\hline \multicolumn{2}{|c|}{ Age } & $45.89 \pm 6.80$ & $47.15 \pm 8.25$ & $47.27 \pm 8.53$ & 0.34 \\
\hline \multirow{2}{*}{ Sex } & Female & $73(48.7)$ & $42(42.9)$ & $45(86.5)$ & \multirow{2}{*}{$<0.001$} \\
\hline & Male & $77(51.3)$ & $56(57.1)$ & $7(13.5)$ & \\
\hline \multicolumn{2}{|c|}{ BMI } & $24.73 \pm 2.98$ & $25.28 \pm 2.77$ & $25.66 \pm 2.69$ & 0.09 \\
\hline \multirow{2}{*}{ Education } & Educated & $131(87.3)$ & $68(69.4)$ & $32(61.5)$ & \multirow{2}{*}{$<0.001$} \\
\hline & Uneducated & $19(12.7)$ & $30(30.6)$ & $20(38.5)$ & \\
\hline \multirow{2}{*}{ Employment } & Employed & $86(57.3)$ & $59(60.2)$ & $20(38.5)$ & \multirow{2}{*}{0.03} \\
\hline & Unemployed & $64(42.7)$ & $39(39.8)$ & $32(61.5)$ & \\
\hline \multicolumn{2}{|c|}{ Duration of diabetes } & - & $3.6 \pm 4.36$ & $6.53 \pm 7.39$ & 0.01 \\
\hline \multicolumn{2}{|c|}{$\mathrm{HbA}_{1 \mathrm{c}}$} & - & $8.78 \pm 2.23$ & $9.19 \pm 2.64$ & 0.30 \\
\hline \multicolumn{2}{|c|}{ PHQ-9 score } & $1.75 \pm 4.15$ & $3.56 \pm 5.63$ & $6.96 \pm 7.49$ & $<0.001$ \\
\hline \multirow{2}{*}{\multicolumn{2}{|c|}{$\begin{array}{c}\text { No depression } \\
\text { Depression }\end{array}$}} & $125(83.3)$ & $65(66.3)$ & $24(46.2)$ & \multirow{2}{*}{$<0.001$} \\
\hline & & $25(16.7)$ & $33(33.7)$ & $28(53.8)$ & \\
\hline \multicolumn{2}{|c|}{ MMSE score } & $28.15 \pm 2.34$ & $26.00 \pm 3.914$ & $25.12 \pm 3.93$ & $<0.001$ \\
\hline \multirow{2}{*}{ Cognitive Impairment } & No Cognitive Impairment & $141(94)$ & $76(77.6)$ & $39(75)$ & \multirow{2}{*}{$<0.001$} \\
\hline & Cognitive Impairment & $9(6)$ & $22(22.4)$ & $13(25)$ & \\
\hline \multicolumn{2}{|c|}{ Physical functioning } & $84.83 \pm 20.26$ & $78.88 \pm 23.97$ & $69.62 \pm 23.76$ & $<0.001$ \\
\hline \multicolumn{2}{|c|}{ Role limitations (physical) } & $73.83 \pm 35.98$ & $61.22 \pm 37.67$ & $49.52 \pm 41.56$ & $<0.001$ \\
\hline \multicolumn{2}{|c|}{ Role limitations (emotional) } & $75.63 \pm 35.31$ & $67.06 \pm 39.34$ & $55.15 \pm 44.74$ & 0.004 \\
\hline \multicolumn{2}{|c|}{ Energy/fatigue } & $69.20 \pm 22.18$ & $64.29 \pm 18.37$ & $56.73 \pm 18.81$ & 0.001 \\
\hline \multicolumn{2}{|c|}{ Emotional well-being } & $76.03 \pm 22.24$ & $70.00 \pm 22.919$ & $62.08 \pm 22.92$ & 0.001 \\
\hline \multicolumn{2}{|c|}{ Social functioning } & $93.67 \pm 16.44$ & $79.49 \pm 33.01$ & $60.15 \pm 38.97$ & $<0.001$ \\
\hline \multicolumn{2}{|c|}{ Pain } & $87.39 \pm 18.52$ & $75.65 \pm 28.95$ & $55.98 \pm 33.58$ & $<0.001$ \\
\hline \multicolumn{2}{|c|}{ General health } & $63.30 \pm 22.26$ & $51.64 \pm 20.27$ & $41.92 \pm 21.85$ & $<0.001$ \\
\hline
\end{tabular}

Data presented as Mean \pm SD or $n(\%){ }^{*}$ value

n-number; BMI-body mass index; FPG-fasting plasma glucose; HbAlc-glycated hemoglobin; PHQ-9-patient health questionnaire; MMSE-mini-mental state examination. 
The likelihood of depression was not significantly associated with duration of T2DM and glycemic control.

Assessment of cognitive function in cases and controls - The mean MMSE score for cases (25.69 \pm 3.93$)$ was lower than controls $28.15 \pm 2.34$, $p=0.000$. The prevalence of cognitive impairment was significantly higher in the cases than in the controls $(35(23.3 \%)$ vs. $9(6 \%), p=0.000)$. In multivariate logistic regression the odds of having cognitive impairment in patients with T2DM was found to be higher than that in the control subjects (aOR 2.86, 95\% CI 1.16-6.99; $p=0.021$ ) (adjusted for age, sex, BMI, education and occupation). Among the 44 (14.66\%) subjects having cognitive impairment, a statistically higher prevalence was observed among the women 33 (75\%) than in men 11 (25\%), $p=0.000$. Of these 44 cognitive impairment subjects, unemployed subjects had higher prevalence 33 (75\%) than those who were employed 11 (25\%), $p=0.000$.

Assessment of Quality of life in cases and controls - Scores for cases were significantly lower than scores for controls in all the domains of SF 36 (Figure 1), suggesting a poorer QoL of diabetics.

Association of type of treatment and depression - Post hoc analysis revealed significantly higher PHQ-9 scores in subjects on insulin therapy (6.96 \pm 7.49$)$ as compared to controls $(1.75 \pm 4.15), p<0.001$ and those receiving OHAs (3.56 \pm 5.63$)$ and, $p<0.001$. Moreover, PHQ-9 scores were significantly higher in subjects receiving OHAs than in healthy subjects, $p=0.026$. Additionally, the prevalence of depression was significantly higher in patients receiving insulin $[27(51.92 \%)]$ as compared to patients on OHAs [33 (35.86\%)], OR 2.127, 95\% CI 1.070-4.228, $p=0.036$.

Association of type of treatment and cognitive impairment - Post hoc analysis revealed significantly lower MMSE scores in patients receiving insulin $(25.12 \pm 3.93)$ and OHAs $(26 \pm 3.91)$ as compared to controls (28.15 \pm 2.34$), p<0.001$ for both. However, there was no significant difference in MMSE scores in patients receiving insulin and OHAs, $p=0.249$. Although, the prevalence of cognitive impairment was higher in insulin receiving group [14 (26.92\%)] than those receiving OHAs [21 (21.43\%)], the difference could not reach the level of significance, $p=0.54$.

Association of type of treatment and QOL - Significantly lower scores were found in physical functioning, energy/fatigue, emotional well-being, social functioning, pain and general health domains of SF-36, in patients receiving insulin as compared to those on OHAs (Figure 2).

\section{DISCUSSION}

Diabetes and depression, two of the world's most serious public health issues, are predicted to be among the top five causes of disease burden by 2030 . The current study found that patients with T2DM had a greater risk of depression than healthy people. Furthermore, depression was

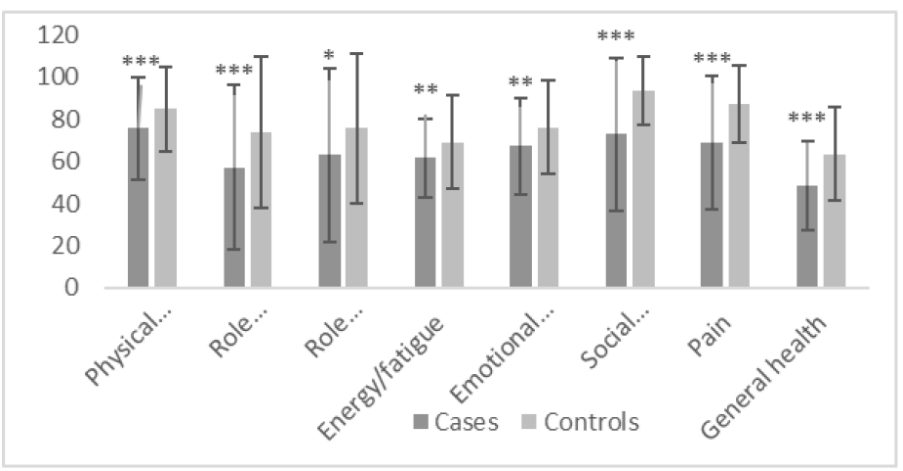

Figure 1: Domain wise SF-36 scores in cases and controls.

${ }^{*} p<0.05 ;{ }^{* *} p<0.01 ;{ }^{* * *} p<0.001$ comapre with that of control.

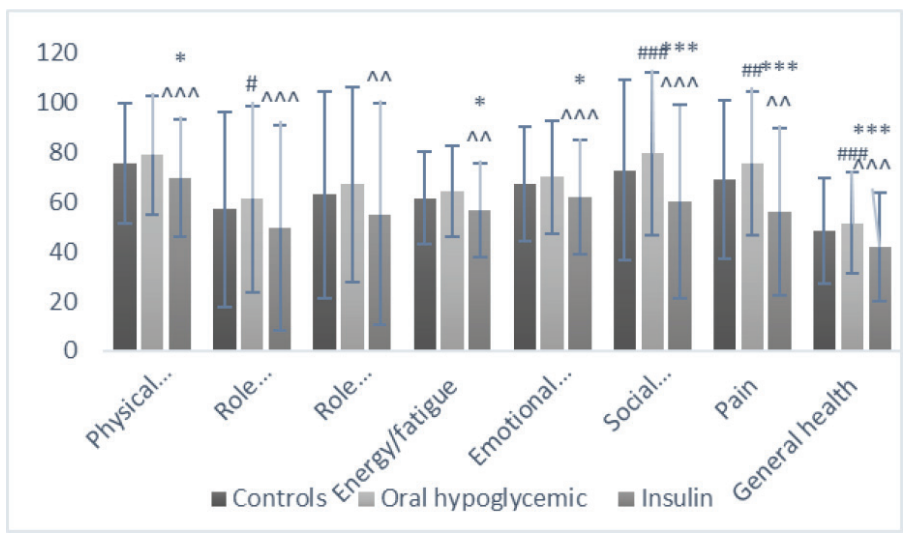

Figure 2: Domain wise SF-36 scores in subjects according to the type of treatment.

${ }^{*} p<0.05$, oral hypoglycemic vs insulin; ${ }^{* *} p<0.01$, oral hypoglycemic vs insulin; ${ }^{* * *} p<0.001$, oral hypoglycemic vs insulin; $\# p<0.05$, controls vs oral

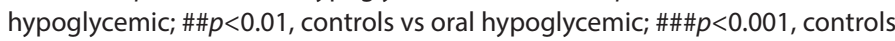
vs oral hypoglycemic; $\wedge p<0.05$, controls vs insulin; $\wedge \wedge p<0.01$, controls vs insulin; $\wedge \wedge \wedge p<0.001$, controls vs insulin

shown to be more common in women and those who were jobless. Clinical trials that have shown comparable outcomes back up these findings. Identical clinical investigations have found that individuals with T2DM had a greater prevalence of depression. A research reported that females have a greater prevalence of depression than males, which is similar to our findings. Furthermore, no link between depression and diabetes duration or HbAlc was discovered. Furthermore, a cross-sectional study indicated that females, those with less education, and those who are unemployed have a greater frequency of depression. ${ }^{23}$

In addition, individuals taking insulin had a greater prevalence of depression than those getting OHAs, according to the current study. Several clinical investigations have found a link between insulin use and the occurrence of depression, which is consistent with our findings. ${ }^{24}$ Several psychological variables have been linked to the increased frequency of depression in insulin-treated individuals. The switch from oral diabetes medications to insulin may have a significant psychological impact on individuals. Insulin treatment may be more difficult for patients than oral medication. Another cause might be injection discomfort, ${ }^{24}$ hypoglycemic episodes, ${ }^{25}$ weight gain ${ }^{25}$ complication of daily life, dietary restrictions, more frequent hospitalization, and the feelings of bad health. Patients may also feel a lack of control over their diabetes, which they see as a factor restricting their social functioning. These psychological factors might explain why those who use insulin have a higher risk of depression.

Diabetes patients exhibited lower MMSE scores than non-diabetic participants in this research. Although MMSE scores were shown to be linked to education, they were not linked to HbAlc levels or illness duration. Clinical investigations have revealed that diabetics had a lower MMSE score than controls, corroborating these findings. A cohort study of T2DM patients revealed a decreased MMSE score, suggesting that T2DM patients should have their cognitive function evaluated on a regular basis. ${ }^{25}$ The cognitive performance of individuals taking OHAs or insulin did not change significantly in this research. These findings are confirmed by earlier research, which found no significant influence on cognitive performance in diabetes individuals in a longitudinal study. Metformin has been shown to preserve verbal learning, working memory, and executive function. Another case-control research found that long-term use of thiazolidinedione, sulfonylureas, or insulin was not 
linked to the development of Alzheimer's disease (AD). However, it was shown that individuals who used metformin for a longer period of time had a slightly increased chance of developing Alzheimer's disease.

Diabetic patients had a lower quality of life than controls, according to the current study. Diabetics had considerably poorer ratings in the majority of SF-36 areas (including role constraints owing to physical health, energy/fatigue, emotional well-being, social functioning, pain, and overall health). In all areas, women scored lower than men, although only physical functioning and pain were shown to be substantially different. These findings are consistent with prior research. A comparable case control research found that diabetics had a poorer quality of life than healthy people. Furthermore, numerous cross-sectional studies have shown that diabetics have poorer QoL ratings. Female patients had considerably worse QoL and mental well-being than male patients, according to a cross-sectional research. ${ }^{26}$ Another cross-sectional study found a strong negative link between females' physical functioning, role emotional, and mental health and males. Females, on the other hand, showed a strong positive connection between physiological discomfort and social functioning.

We also found that patients who received insulin had a lower quality of life than those who received OHAs. Physical functioning, social functioning, and pain were all considerably decreased in insulin-treated individuals. This conclusion is confirmed by a number of previous clinical investigations that have linked insulin usage to decreased quality of life. ${ }^{26}$ Insulin usage was linked to a considerable drop in physical functioning, greater body discomfort, and a decline in overall health and social functioning. Side effects from insulin treatment that influence the scheduling and control of daily activities, the fear of weight gain, and the impact of insulin treatment on the social environment have all been linked to lower QoL in insulin therapy patients. ${ }^{27}$ Alternative explanations for these findings in such individuals include the disease's prolonged duration, dissatisfaction following the failure of oral medicines, or the emergence of diabetes-related complications and the consequent need to begin insulin therapy. ${ }^{27}$

There are a few limitations to the study that should be addressed. Several types of data were self-reported or retrieved from patient medical records, including T2DM duration and education status. This may have resulted in memory bias. No causal link could be drawn because this was a cross-sectional research. Finally, the sample size was limited, thus it's possible that not all diabetes patients were included.

\section{CONCLUSION}

Depression, cognitive impairment, and poor quality of life are more common in T2DM patients than in healthy people. Furthermore, patients who receive insulin have a higher rate of depression and a lower quality of life than those who receive OHAs. Routine screening of T2DM patients is thus recommended in order to avoid and limit any additional psychological problems. T2DM patients should receive proper education and follow-up, which may help them avoid impairment. Because of the negative effects of insulin on mood and quality of life, diabetic individuals who are on insulin may require psychological assistance. Furthermore, prospective studies with a high sample size are needed to further understand the relationship between treatment regimen, depression, cognitive function, and QoL in T2DM patients.

\section{ACKNOWLEDGEMENT}

The authors are thankful to University Grants Commission, India for providing fellowship to Dr. Rizwana.

\section{CONFLICT OF INTEREST}

The authors declare no conflict of interest.

\section{REFERENCES}

1. Parveen R, Agarwal NB, Kaushal N, Mali G, Raisuddin S. Efficacy and safety of canagliflozin in type 2 diabetes mellitus: systematic review of randomized controlled trials. Expert Opin Pharmacother. 2016;17(1):105-15. doi: 10.1517/14656566.2016.1109629, PMID 26650511.

2. Herath PM, Cherbuin N, Eramudugolla R, Anstey KJ. The effect of diabetes medication on cognitive function: evidence from the PATH through life study. BioMed Res Int. 2016;2016:Article ID 7208429, 7 pages. doi: 10.1155/2016/7208429, PMID 27195294.

3. IDF diabetes atlas. International Diabetes Federation - Home; 2019. $9^{\text {th }}$ ed. Available from: idf.org

4. Imran KM, Ismail AA, Naing L, Wan Mohamad WB. Type 2 diabetes mellitus patients with poor glycaemic control have lower quality of life scores as measured by the Short Form-36. Singapore Med J. 2010;51(2):157-62. PMID 20358156.

5. Bai X, Liu Z, Li Z, Yan D. The association between insulin therapy and depression in patients with type 2 diabetes mellitus: A meta-analysis. BMJ, (Open) 2018;8(11):e020062. doi: 10.1136/bmjopen-2017-020062, PMID 30498035.

6. Wang J, He M. Levels of serum brain-derived neurotrophic factor in type 2 diabetes mellitus patients with and without depressive symptoms. Acta Biochim Biophys Sin (Shanghai). 2015:47(2):137-8. doi: 10.1093/abbs/gmu117 PMID 25520175.

7. Siddiqui S. Depression in type 2 diabetes mellitus--a brief review. Diabetes Metab Syndr. 2014;8(1):62-5. doi: 10.1016/j.dsx.2013.06.010, PMID 24661762.

8. Gorska-Ciebiada M, Saryusz-Wolska M, Ciebiada M, Loba J. Mild cognitive impairment and depressive symptoms in elderly patients with diabetes: Prevalence, risk factors, and comorbidity. J Diabetes Res. 2014;2014:179648. doi: $10.1155 / 2014 / 179648$

9. Berge LI, Riise T, Tell GS, Iversen MM, Østbye T, Lund A, et al. Depression in persons with diabetes by age and antidiabetic treatment: A cross-sectional analysis with data from the Hordaland Health Study. PLOS ONE. 2015;10(5):e0127161. doi: 10.1371/journal.pone.0127161, PMID 26010615.

10. Habtewold TD, Alemu SM, Haile YG. Sociodemographic, clinical, and psychosocial factors associated with depression among type 2 diabetic outpatients in Black Lion General Specialized Hospital, Addis Ababa, Ethiopia: A crosssectional study. BMC Psychiatry. 2016;16:103. doi: 10.1186/s12888-016-0809-6, PMID 27083154

11. Joseph N, Unnikrishnan B, Raghavendra Babu YP, Kotian MS, Nelliyanil M Proportion of depression and its determinants among type 2 diabetes mellitus patients in various tertiary care hospitals in Mangalore city of South India. Indian J Endocrinol Metab. 2013;17(4):681-8. doi: 10.4103/2230-8210.113761, PMID 23961486.

12. Chen $\mathrm{RH}$, Zhao XH, Gu Z, Gu PL, Zhou B, Zhu ZH, et al. Serum levels of 25-hydroxyvitamin $D$ are associated with cognitive impairment in type 2 diabetic adults. Endocrine. 2014;45(2):319-24. doi: 10.1007/s12020-013-0041-9, PMID 23982512.

13. Karan NS. Assessment of the cognitive status in diabetes mellitus. J Clin Diagn Res. 2012;6(10):1658-62. doi: 10.7860/JCDR/2012/4837.2649, PMID 23373022

14. Ebady SA, Arami MA, Shafigh MH. Investigation on the relationship between diabetes mellitus type 2 and cognitive impairment. Diabetes Res Clin Pract. 2008;82(3):305-9. doi: 10.1016/j.diabres.2008.08.020, PMID 18848366

15. Moore EM, Mander AG, Ames D, Kotowicz MA, Carne RP, Brodaty $H$, et al. Increased risk of cognitive impairment in patients with diabetes is associated with metformin. Diabetes Care. 2013;36(10):2981-7. doi: 10.2337/dc13-0229 PMID 24009301.

16. Espeland MA, Miller ME, Goveas JS, Hogan PE, Coker LH, Williamson J, et al. Cognitive function and fine motor speed in older women with diabetes mellitus: results from the women's health initiative study of cognitive aging. J Womens Health (Larchmt). 2011;20(10):1435-43. doi: 10.1089/jwh.2011.2812, PMID 21819251.

17. Didarloo A, Alizadeh M. Health-related quality of life and its determinants among women with diabetes mellitus: A cross-sectional analysis. Nurs Midwif Stud. 2016;5(1):e28937. doi: 10.17795/nmsjournal28937, PMID 27331054.

18. Thommasen HVZ, W. Health-related quality of life and type 2 diabetes: A study of people living in the Bella Coola Valley. BC Med J. 2006;48:272-8.

19. Spasic A, Radovanovic RV, Đordevic AC, Stefanovic N, Cvetkovic T. Quality of life in Type 2 diabetic patients. Acta Fac Med Naissensis. 2014;31(3):193-200. do: 10.2478/afmnai-2014-0024.

20. Javanbakht M, Abolhasani F, Mashayekhi A, Baradaran HR, Jahangiri noudeh $Y$. Health related quality of life in patients with type 2 diabetes mellitus in Iran: a national survey. PLOS ONE. 2012;7(8):e44526. doi: 10.1371/journal. pone.0044526, PMID 22952989.

21. Dabrowski M, FilipW, Huc B. Association between insulin therapy and quality of life in diabetes. J Pre Clin Clin Res. 2017;11(1):10-4. doi: 10.26444/jpccr/75145. 
22. Sepúlveda E, Poínhos R, Constante M, Pais-Ribeiro J, Freitas P, Carvalho D. Health-related quality of life in type 1 and type 2 diabetic patients in a Portuguese central public hospital. Diabetes Metab Syndr Obes. 2015;8:219-26. doi: 10.2147/DMSO.S80472, PMID 25995647.

23. Sweileh WM, Abu-Hadeed HM, Al-Jabi SW, Zyoud SH. Prevalence of depression among people with type 2 diabetes mellitus: a cross sectional study in Palestine. BMC Public Health. 2014;14:163. doi: 10.1186/1471-2458-14-163, PMID 24524353.

24. Noh JH, Park JK, Lee HJ, Kwon SK, Lee SH, Park JH, et al. Depressive symptoms of type 2 diabetics treated with insulin compared to diabetics taking oral anti-diabetic drugs: a Korean study. Diabetes Res Clin Pract. 2005;69(3):243-8. doi: 10.1016/j.diabres.2004.10.009, PMID 16046024.

25. Derakhshanpour F, Vakili MA, Farsinia M, Mirkarimi K. Depression and quality of life in patients with Type 2 diabetes. Iran Red Crescent Med J. 2015;17(5):e27676. doi: 10.5812/ircmj.17(5)2015.27676, PMID 26082854.

26. Mohammadi S, Karim N, Abd. Talib R, Amani R. Evaluation of quality of life among type 2 diabetes patients. Int J Community Med Public Health:51-6. doi: 10.18203/2394-6040.ijcmph20151485.

27. Kodl CT, Seaquist ER. Cognitive dysfunction and diabetes mellitus. Endocr Rev. 2008;29(4):494-511. doi: 10.1210/er.2007-0034, PMID 18436709.

Article History: Submission Date : 07-07-2021; Revised Date : 08-08-2021; Acceptance Date : 29-08-2021.

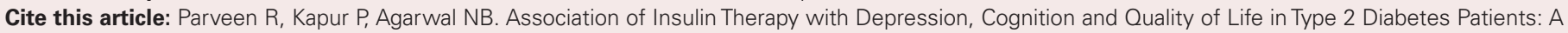
Cross-sectional Study. Int. J. Pharm. Investigation. 2021;11(3):327-32. 